\title{
ULTRAPRODUCT INVARIANT LOGICS
}

\author{
J. SGRO ${ }^{1}$
}

\begin{abstract}
In this paper we give a construction of logics via the property of being preserved from the models to their ultraproduct. Specific examples are given which include some cardinality quantifiers.
\end{abstract}

We will assume that the reader is familiar with basic model theory including ultraproducts, Chang and Keisler [4], and abstract logics, confer Barwise [1].

Definition. ( $\mathfrak{A}, \vec{q})$ is called a generalized model if $\vec{q}(i) \subseteq \mathscr{P}\left(A^{m(i)}\right)$ for each $i$ (where $m(i) \in \omega$ and the function $m$ is called the type of the model).

Take Mod to be a class of generalized models of the same type closed under restriction and expansion of languages.

Let $\mathrm{Seq}_{\kappa}(\mathrm{Mod})$ be the class of all sequences of length $\kappa$ of elements of Mod, $\operatorname{Ult}(\kappa)$ be the set of ultrafilters on $\kappa$, and $\operatorname{Seq}(\operatorname{Mod})=\cup_{\kappa} \operatorname{Seq}_{\kappa}(\mathrm{Mod})$ and Ult $=U_{\kappa} \operatorname{Ult}(\kappa)$.

Definition. An ultraproduct on Mod is a partial function ULT from $\cup_{\kappa}$ Seq $_{\kappa}($ Mod $) \times \operatorname{Ult}(\kappa)$ into Mod.

We say that the pair $\langle$ Mod, ULT $\rangle$ is closed under $\kappa$-ultraproducts if Seq $_{\kappa}($ Mod) $\times \operatorname{Ult}(\kappa) \subseteq$ Dom ULT. If $\langle$ Mod, ULT〉 is closed under $\kappa$-ultraproducts for all cardinals $\kappa$ then it is said to be closed under ultraproducts.

Let $\left\langle\mathfrak{L}^{2}, F_{\mathfrak{Q}^{2}}\right\rangle$ be the second-order logic with additional quantification over the generalized domains. That is:

(a) the usual clauses for generalized first-order logics,

(b) if $t$ is a term and $X_{m}^{\vec{q}(i)}$ is a generalized variable then $t \in X_{m}^{\vec{q}(i)}$ is a formula and $(\mathfrak{A}, \vec{q}) \vDash_{\mathfrak{e}^{2 t}} \in X_{m}^{\vec{q}(i)}[s]$ if and only if $s(t) \in s\left(X_{m}^{\vec{q}(i)}\right)$ where $s\left(X_{m}^{\vec{q}(i)}\right) \in \vec{q}(i)$,

(c) if $\varphi(R)$ (or $\varphi(f)$ ) are formulas where $R(f)$ is a relation (function) variable then $\exists R \varphi(R)(\exists f \varphi(f))$ is a formula. $\exists R \varphi(R)$ and $\exists f \varphi(f)$ have the usual secondorder interpretation,

(d) if $\varphi\left(X^{\vec{q}(i)}\right)$ is a formula then so is $\exists X_{m}^{\vec{q}(i)} \varphi\left(X_{m}^{\vec{q}(i)}\right)$. (趾, $\left.\vec{q}\right) \vDash_{\mathfrak{R}^{2}} \exists X_{m}^{\vec{q}(i)} \varphi\left(X_{m}^{\vec{q}(i)}\right)$ if and

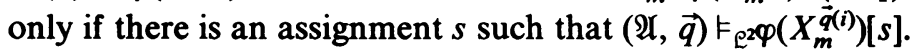

Let Mod and ULT be given.

Definition. A sentence $\varphi$ in the language $L$ of $L^{2}$ is called invariant if and only if for each $\left\langle\left\{\left(\mathfrak{A}_{\alpha}, \vec{q}_{\alpha}\right)\right\}_{\alpha<\kappa}, D\right\rangle \in \operatorname{Dom}$ ULT (each $\left(\mathfrak{A}_{\alpha}, q_{\alpha}\right)$ is an L-model) $\left\{\alpha \mid\left(\mathfrak{A}_{\alpha}, q_{\alpha}\right) \vDash_{\mathfrak{R}^{2} \varphi} \varphi \in D\right.$ if and only if $\operatorname{ULT}\left(\left\{\left(\mathfrak{A}_{\alpha}, q_{\alpha}\right)\right\}_{\alpha \in \kappa}, D\right) \vDash_{\mathbb{R}^{2}} \varphi$.

Let $I$ (Mod, ULT) be the sublogic of $\mathfrak{L}^{2}$ consisting of the invariant sentences.

Let $\mathrm{Ult}^{*}(\kappa)$ be the class of regular ultrafilters over $\kappa$.

Received by the editors July $25,1978$.

AMS (MOS) subject classifications (1970). Primary 02H99; Secondary 02C99, 02 G99.

'This research was partially supported by NSF Grant MCS 77-04131.

(C) 1980 American Mathematical Society 0002-9939/80/0000-0378/\$02.00 
Theorem. If $\operatorname{Seq}_{\kappa}(\mathrm{Mod}) \times \operatorname{Ult}^{*}(\kappa) \subseteq$ Dom ULT then I(Mod, ULT) is $(\omega, \kappa)-$ compact. In fact it satisfies a $\kappa$ version of the Kos ultraproducts theorem.

Proof. Straightforward by the definition of I(Mod, ULT).

THEOREM. Let ULT commute with restriction. Then I(Mod, ULT) has the Souslin-Kleene Property (i.e. complementary PC $(L)$ classes are $E C(L)$ classes).

REMARK. For ULT to commute with restriction we mean that if $\operatorname{ULT}\left(\left\{\left(\mathfrak{A}_{\alpha}, q_{\alpha}\right)\right\}, D\right)=(\mathfrak{B}, r)$ then $(\mathfrak{B} \uparrow L, r)=\operatorname{ULT}\left(\left\{\left(\mathfrak{A}_{\alpha} \uparrow L, q_{\alpha}\right)\right\}, D\right)$.

Proof. Let $\theta_{0}$ be a sentence of $L_{0}, \theta_{1}$ of $L_{1}$,

$$
\begin{aligned}
& \Omega=\left\{(\mathfrak{U} \uparrow L, \vec{q}) \mid(\mathfrak{A}, \vec{q}) \vDash \theta_{0}\right\}, \\
& \Omega^{c}=\left\{(\mathfrak{A} \uparrow L, \vec{q}) \mid(\mathfrak{A}, q) \vDash \theta_{1}\right\} .
\end{aligned}
$$

We need to show that $\Omega$ is $E C\left(L_{0} \cap L_{1}\right)$.

Define $\vec{\exists} \theta_{i}$ to be the sentence obtained from $\theta_{i}$ by replacing all the relation and function symbols of $L_{i}-L_{0} \cap L_{1}$ by variables and existentially quantifying over them.

We claim that $\vec{\exists} \theta_{i}$ is an $L_{0} \cap L_{1}$-invariant sentence. Suppose $\left\{\alpha \mid\left(\mathfrak{A}_{\alpha}, q_{\alpha}\right) \vDash \vec{\exists} \theta_{i}\right\}$ $\in D$. Then there are $\vec{R}_{\alpha}, \vec{f}_{\alpha}$ such that $\left\{\alpha \mid\left(\hat{U}_{\alpha}, \vec{R}_{\alpha}, \vec{f}_{\alpha}, \vec{q}\right) \vDash \theta_{i}\right\} \in D$. Hence $\operatorname{ULT}\left(\left\{\left(\mathfrak{A}_{\alpha}, \vec{R}_{\alpha}, \vec{f}_{\alpha}, \vec{q}_{\alpha}\right)\right\}_{\alpha \in \kappa}, D\right) \vDash \theta_{i}$.

Using commutativity we get that $\operatorname{ULT}\left(\left\{\left(\mathfrak{A}_{\alpha}, q_{\alpha}\right)\right\}, D\right) \vDash \vec{\exists} \theta_{i}$. The other direction is analogous using the observation that $\left\{\alpha \mid\left(\mathfrak{A}_{\alpha}, q_{\alpha}\right) \vDash \vec{\exists} \theta_{i}\right\} \notin D$ if and only if $\left\{\alpha \mid\left(\mathfrak{A}_{\alpha}, q_{\alpha}\right) \vDash \vec{\exists} \theta_{i-1}\right\} \in D$ where $i-1=0$ if $i=1, i-1=1$ if $i=0$.

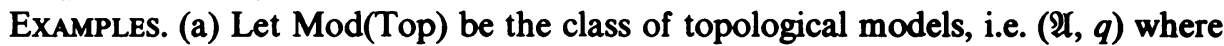
$q$ is a topology on $A$, and ULT(Top) the full topological ultraproduct defined in Sgro [9]. I(Mod(Top), ULT(Top)) is compact since

$$
\operatorname{Seq}(\operatorname{Mod}(T o p)) \times \text { Ult } \subseteq \text { Dom ULT(Top). }
$$

We also know that I(Mod(Top), ULT(Top)) has the Souslin-Kleene Property because ULT(Top) commutes with restriction. In fact, because $\mathcal{L}^{\text {top }}$ (confer Sgro [11]) is invariant and maximal with respect to a Kos ultraproducts theorem, we have $I\left(\right.$ Mod(Top), ULT(Top)) $=\mathfrak{L}^{\text {top }}$.

(b) Let $\kappa$ be a weakly compact cardinal, i.e. $\kappa \rightarrow(\kappa)_{\gamma}^{n}$ for all $\gamma<\kappa$ and $n \in \omega$. Take Mod(w.c.) to be the class of models, $(\mathfrak{A}, q)$, where $q$ is the set of subsets of $A$ of cardinality greater than or equal to $\kappa$. If $D$ is a uniform ultrafilter on $\gamma<\kappa$, then $\mathrm{ULT}($ w.c. $)\left(\left\{\left(\mathfrak{A}_{\alpha}, q_{\alpha}\right)\right\}_{\alpha<\gamma}, D\right)$ is $\left(\Pi_{D} \mathfrak{A}_{\alpha}, q^{*}\right)$ where $q^{*}$ is the collection of subsets of $\Pi_{D} A_{\alpha}$ of cardinality greater than or equal to $\kappa$. $\left(\Pi_{D} \mathfrak{A}_{\alpha}\right.$ is the usual first-order ultraproduct.)

Because ULT(w.c.) commutes with restriction and $\operatorname{Seq} \operatorname{Mod}(w . c.) \times \operatorname{Ult}^{*}(\gamma) \subseteq$ Dom ULT(w.c.) for all $\gamma<\kappa$ we know that $I(\operatorname{Mod}(w . c),. U L T(w . c)$.$) is (\omega, \gamma)$-compact for $\gamma<\kappa$ and has the Souslin-Kleene Property.

Taking $\mathcal{L}\left(Q_{\kappa}^{<\omega}\right)$ to be the Malitz-Magidor quantifier under the $\kappa$ interpretation, confer [7], we claim that $\mathcal{L}\left(Q_{\kappa}^{<\omega}\right) \lesseqgtr I(\operatorname{Mod}(w . c),. \mathrm{ULT}(w . c)$.$) .$

The inequality is straightforward because by $[6] \mathcal{L}\left(Q_{x}^{<\omega}\right)$ does not have the Souslin-Kleene Property. 
We need only show that each $Q_{x_{1}, \ldots, x_{n}}^{n} \varphi\left(x_{1}, \ldots, x_{n}\right)$ is equivalent to an invariant sentence. It is easy to see that the following formula of $\mathfrak{L}^{2}$ is equivalent to $Q_{x_{1}, \ldots, x_{n}}^{n} \varphi\left(x_{1}, \ldots, x_{n}\right)$,

$\varphi^{*}=\exists X_{1}^{q} \forall x_{1}, \ldots, x_{n}\left(\bigvee_{i \neq j} x_{i}=x_{j} \vee \bigvee_{i} x_{i} \notin X_{1}^{q} \vee \underset{\sigma \text { perm. }}{\bigwedge_{\sigma(1)}} \varphi\left(x_{o(1)}, \ldots, x_{\sigma(n)}\right)\right)$.

We claim that it is invariant if $\varphi\left(x_{1}, \ldots, x_{n}\right)$ is. If $\left\{\alpha \mid\left(\mathfrak{A}_{\alpha}, q_{\alpha}\right) \vDash \varphi^{*}\right\} \in D$ then $\operatorname{ULT}($ w.c. $)\left(\left\{\left(\mathfrak{A}_{\alpha}, q_{\alpha}\right)\right\}, D\right) \vDash \varphi^{*}$ is straightforward because existential sentences go up. So suppose that ULT(w.c.) $\left(\left\{\left(\mathfrak{A}_{\alpha}, q_{\alpha}\right)\right\}, D\right) \vDash \varphi^{*}$. That is, there is a subset $X$ of $\Pi_{D} A_{\alpha}$ of cardinality $\kappa$ (which we will well-order by $<$ ) such that for each $a_{1}<\cdots<a_{n} \in X$ we have $\left(\Pi_{D} \mathfrak{A}_{\alpha}, q^{*}\right) \vDash \bigwedge_{\sigma} \varphi\left(a_{\sigma(1)}, \ldots, a_{\sigma(n)}\right)$. Hence by our assumption

$$
\left\{\alpha \mid\left(\mathfrak{A}_{\alpha}, q_{\alpha}\right) \vDash \bigwedge_{\sigma} \varphi\left(a_{\sigma(1)}(\alpha), \ldots, a_{\sigma(n)}(\alpha)\right)\right\} \in D .
$$

Define $H:[X]^{n} \rightarrow D$ as follows: if $\left\{a_{1}<\cdots<a_{n}\right\} \in[X]^{n}$ then

$$
\begin{aligned}
H\left(\left\{a_{1}<\right.\right. & \left.\left.\cdots<a_{n}\right\}\right) \\
& =\left\{\alpha \mid\left(\mathfrak{A}_{\alpha}, q_{\alpha}\right) \vDash \bigwedge_{\sigma} \varphi\left(a_{\sigma(1)}(\alpha), \ldots, a_{\sigma(n)}(\alpha)\right) \wedge \bigwedge_{i \neq j} a_{i}(\alpha) \neq a_{j}(\alpha)\right\}
\end{aligned}
$$

which is in $D$ by (*).

Because $\kappa$ is weakly compact and $|D|<\kappa$ we have that there is a $Y \subseteq X$ such that $H(Y)=\mathcal{Q}$ and $\mathcal{Q} \in D$.

Take $\alpha \in \mathcal{U}$. Then we know that for every $a_{1}<\cdots<a_{n} \in Y$

$$
\left(\mathfrak{A}_{\alpha}, q_{\alpha}\right) \vDash \bigwedge_{\sigma} \varphi\left(a_{\sigma(1)}(\alpha), \ldots, a_{\sigma(n)}(\alpha)\right) \wedge \bigwedge_{i \neq j} a_{i}(\alpha) \neq a_{j}(\alpha) .
$$

So $Y_{\alpha}=\{a(\alpha) \mid a \in Y\}$ has cardinality $\kappa$ and

$$
\begin{aligned}
\mathscr{U} \subseteq\left\{\alpha \mid\left(\mathfrak{A}_{\alpha}, q_{\alpha}\right) \vDash \forall x_{1}, \ldots, x_{n}\left(\bigvee_{i \neq j} x_{i}\right.\right. & =x_{j} \vee \bigvee_{i} x_{i} \notin Y_{\alpha} \\
& \left.\left.\vee \bigwedge_{\sigma} \varphi\left(x_{\sigma(1)}, \ldots, x_{\sigma(n)}\right)\right)\right\} .
\end{aligned}
$$

Hence $\left\{\alpha \mid\left(\mathfrak{A}_{\alpha}, q_{\alpha}\right) \vDash \varphi^{*}\right\} \in D$.

Question. Is $\Delta\left(\mathcal{L}\left(Q_{\kappa}^{<\omega}\right)\right)<I(\operatorname{Mod}($ w.c.), ULT(w.c.))) Confer [6].

Question. What is the relationship between $\mathfrak{L}^{\text {neg }}$ and 1 ? Confer [2].

(c) Let $\operatorname{Mod}\left(\omega_{1}\right)$ be the class of $\omega_{1}$-standard models as in Keisler [5], i.e., ( $(\mathfrak{A}, q)$ where $q$ is the set of uncountable subsets of $A$. We will define an ultraproduct $\operatorname{ULT}\left(\omega_{1}\right)$ on $\operatorname{Mod}\left(\omega_{1}\right)$ such that $\mathscr{L}\left(Q_{\omega_{1}}\right)$ will be invariant. Confer [5].

Let $D$ be an ultrafilter on a countable set and $\left\{\left(\mathfrak{A}_{i}, q_{i}\right)\right\}_{i \in \omega}$ a sequence of $\omega_{1}$-standard models. If $D$ is principal then $\operatorname{ULT}\left(\left\{\left(\mathfrak{A}_{i}, q_{i}\right)\right\}_{i \in \omega}, D\right)=\left(\mathfrak{A}_{j}, q_{j}\right)$ where $\{j\}$ generates $D$.

If $D$ is nonprincipal then it is $\omega_{1}$-good, confer [4]. Hence $\Pi_{D}\left(\mathfrak{A}_{i}, q_{i}\right)$ (the standard many-sorted ultraprouct) is $\omega_{1}$-saturated with respect to $\mathcal{L}\left(Q_{\omega_{1}}\right)$. Now by Keisler [5] there is an $\omega_{1}$-standard model $(\mathfrak{B}, r)$, elementarily equivalent to $\Pi_{D}\left(\mathfrak{A}_{i}, q_{i}\right)$.

Thus if $r^{*}$ is the $\mathfrak{L}\left(Q_{\omega}\right)$-definable-over-( $(B, r)$ subsets of $B$ in $r$ we have an $\mathcal{L}\left(Q_{\omega_{1}}\right)$-elementary embedding $h$ of $\left(\mathfrak{B}, r^{*}\right)$ into $\Pi_{D}\left(\mathscr{U}_{i}, q_{i}\right)$. Letting $h(r)$ be the 
uncountable subsets of $h(B)$ we have $(h(\mathscr{B}), h(r)) \equiv_{\mathscr{e}\left(Q_{\omega}\right)} \Pi_{D}\left(\mathfrak{A}_{i}, q_{i}\right)$ and $h(\mathfrak{B}) \prec$ $\Pi_{D} \mathfrak{A}_{i}$. Define ULT $\left(\operatorname{Mod}\left(\omega_{1}\right), D\right)=(h(\mathfrak{B}), h(r))$. Then $\mathcal{E}\left(Q_{\omega_{1}}\right)<I\left(\operatorname{Mod}\left(\omega_{1}\right)\right.$, $\left.\operatorname{ULT}\left(\omega_{1}\right)\right)$ which is countably compact.

Question. How strong is $I\left(\operatorname{Mod}\left(\omega_{1}\right), \operatorname{ULT}\left(\omega_{1}\right)\right)$ for various choices of $(\mathfrak{B}, r)$ ?

(d) Assume $\diamond_{\omega_{1}}$, confer [7]. Using the completeness theorem proved in [7] for $\mathcal{L}\left(Q_{\omega_{1}}^{<\omega}\right)$ we define an ultraproduct on $\operatorname{Mod}\left(\omega_{1}\right)$ such that $\mathcal{L}\left(Q_{\omega_{1}}^{<\omega}\right)$ is invariant in an analogous fashion to (c). Also we can treat $\Delta\left(\mathcal{L}\left(Q_{\omega_{1}}\right)\right)$ and $\Delta\left(\mathcal{L}\left(Q_{\omega_{1}}^{<\omega}\right)\right)$ analogously.

RemarKs. We would like to note that in example (a) we could have taken any invariant logic (in the sense of [11]) and had the analogous result: i.e. $I(\operatorname{Mod}(\mathscr{F})$, $\operatorname{ULT}(\mathscr{F}))=\mathfrak{L}^{\mathscr{F}}$. Also our choice of $\mathfrak{L}^{2}$ was somewhat arbitrary and we would like to point out that many other choices, e.g. logics with higher order variables would be equally good, see [8], which would have similar questions associated with them.

\section{REFERENCES}

1. K. J. Barwise, Axioms for abstract model theory, Ann. Math. Logic 7 (1974), 221-265.

2. K. J. Barwise, M. Kaufmann and M. Makkai, Stationary logic, Ann. Math. Logic 13 (1978), 171-224.

3. K. Bruce, Ideal models and not so ideal problems, J. Symbolic Logic 43 (1978), 304-321.

4. C. C. Chang and H. J. Keisler, Model theory, North-Holland, Amsterdam, 1973.

5. H. J. Keisler, Logic with the quantifier "there exists uncountably many", Ann. Math. Logic 1 (1970), 1-93.

6. J. Makowsky, S. Shelah and J. Stavi, $\Delta$-Logics and generalized quantifiers, Ann. Math. Logic 10 (1976), 155-192.

7. M. Magidor and J. Malitz, Compact extension of $L(Q)$, Part la, Ann. Math. Logic 11 (1977), 217-261.

8. J. Malitz and M. Rubin, Notices Amer. Math. Soc. 25 (1978), Abstracts 78T-E51, p. A-442, 78T-E52, p. A-443 and 78T-E53, p. A-443.

9. J. Sgro, Completeness theorems for topological models, Ann. Math. Logic 11 (1977), 173-193.

10. Completeness theorems for continuous functions and product topologies, Israel J. Math. 25 (1976), 249-272.

11. __ Maximal logics, Proc. Amer. Math. Soc. 63 (1977), 291-298.

12. 99-112.

Department of Mathematics, Institute for Advanced Study, Princeton, New Jersey 08540

Current address: 12323 Brookshire Avenue, Downey, California 90242 